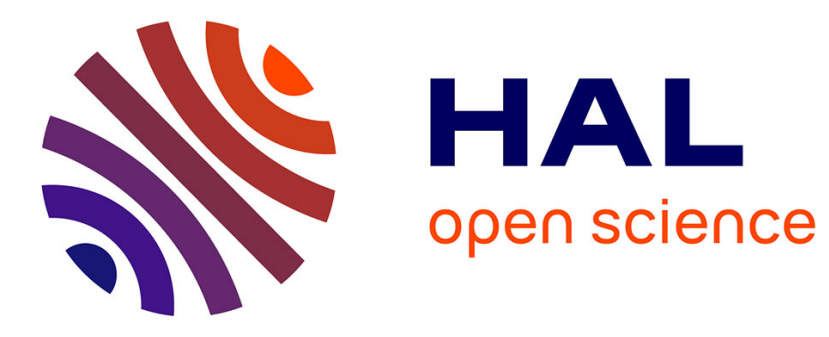

\title{
Head Tracking and Flagellum Tracing for Sperm Motility Analysis
}

\author{
Huei-Fang Yang, Xavier Descombes, Sylvain Prigent, Grégoire Malandain, \\ Xavier Druart, Franck Plouraboué
}

\section{- To cite this version:}

Huei-Fang Yang, Xavier Descombes, Sylvain Prigent, Grégoire Malandain, Xavier Druart, et al.. Head Tracking and Flagellum Tracing for Sperm Motility Analysis. ISBI - International Symposium on Biomedical Imaging, Apr 2014, Beijing, China. 10.1109/ISBI.2014.6867871 • hal-00909868v2

\section{HAL Id: hal-00909868 https://hal.inria.fr/hal-00909868v2}

Submitted on 13 Dec 2013

HAL is a multi-disciplinary open access archive for the deposit and dissemination of scientific research documents, whether they are published or not. The documents may come from teaching and research institutions in France or abroad, or from public or private research centers.
L'archive ouverte pluridisciplinaire HAL, est destinée au dépôt et à la diffusion de documents scientifiques de niveau recherche, publiés ou non, émanant des établissements d'enseignement et de recherche français ou étrangers, des laboratoires publics ou privés. 


\title{
HEAD TRACKING AND FLAGELLUM TRACING FOR SPERM MOTILITY ANALYSIS
}

\author{
H.-F. Yang ${ }^{\dagger}$, X. Descombes ${ }^{\ddagger}$, S. Prigent ${ }^{\S}$, G. Malandain ${ }^{\ddagger}$, X. Druart ${ }^{\ddagger}$, F. Plouraboué ${ }^{\|}$
}

\begin{abstract}
Sperm quality assessment plays an essential role in human fertility and animal breeding. Manual analysis is time-consuming and subject to intra- and inter-observer variability. To automate the analysis process, as well as to offer a means of statistical analysis that may not be achieved by visual inspection, we present a computational framework that tracks the heads and traces the tails for analyzing sperm motility, one of the most important attributes in semen quality evaluation. Our framework consists of 3 modules: head detection, head tracking, and flagellum tracing. The head detection module detects the sperm heads from the image data, and the detected heads are the inputs to the head tracking module for obtaining the head trajectories. Finally, a flagellum tracing algorithm is proposed to obtain the flagellar beat patterns. Our framework aims at providing both the head trajectories and the flagellar beat patterns for quantitatively assessing sperm motility. This distinguishes our work from other existing methods that analyze sperm motility based merely on the head trajectories. We validate our framework using two confocal microscopy image sequences of ram semen samples that were imaged at two different conditions, at which the sperms behave differently. The results show the effectiveness of our framework.
\end{abstract}

Index Terms - object detection, object tracking, flagellum tracing, sperm motility analysis, confocal microscopy

\section{INTRODUCTION}

Sperm quality assessment plays an important role in human fertility and animal breeding. One of the most important attributes for evaluating semen quality is sperm motility, according to the World Health Organization (WHO) report [1]. When performed manually, semen analysis based on sperm motility is labor-intensive and subject to intra- and inter-observer variability. Computer-assisted sperm analysis (CASA) systems, in contrast, provide rapid and objective semen fertility assessment. In addition, they also offer a means of statistical analysis that may not be achieved by visual assessment. Hence, automated sperm motility analysis systems are highly desirable.

To analyze the sperm motility, algorithms that obtain the trajectories of sperm heads have been developed in the CASA systems, which are mainly for evaluating the velocity of individual sperms. Such algorithms are based on template matching [2], particle filtering [3], and motion template [4]. While great effort has been made to

\footnotetext{
* THIS WORK IS SUPPORTED BY ANR MOTIMO PROJECT.

${ }^{\dagger}$ Univ. Nice Sophia Antipolis, CNRS, I3S, UMR 7271, 06900 Sophia Antipolis, France.

${ }^{\ddagger}$ INRIA, 06900 Sophia Antipolis, France.

$\S$ Univ. Nice Sophia Antipolis, CNRS, INSERM, iBV, UMR 7277, 06100 Nice, France.

ๆINRA, CNRS-Université de Tours-Haras Nationaux, 37380 Nouzilly, France.

"IMFT UMR 5502 CNRS/INPT/UPS, 31400 Toulouse, France. $\star$ H.-F. Yang, X. Descombes, S. Prigent, and G. Malandain are in the team MORPHEME, INRIA/I3S/IBV, 06903 Sophia Antipolis, France.
}

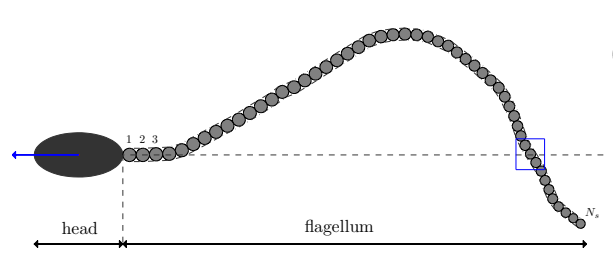

(a)

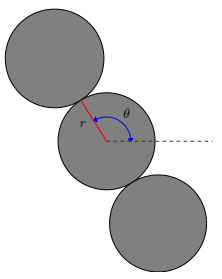

(b)
Fig. 1. Representation of a spermatozoon. (a) A spermatozoon consists of a head and a flagellum. The head is represented by an ellipse. The direction of its major axis indicates the direction of sperm movement (the blue arrow). The red point is the head center. The flagellum is made up of a sequence of $N_{s}$ circles. (b) Close-up of the circles in the bounding box in (a) is shown. Each circle is parameterized by a radius $(r)$ and an angle $(\theta)$.

develop head tracking algorithms, relatively little attention has been given to obtaining the shapes of flagellar beat. In biology, it is established that the shapes of flagellar beat determine the moving path of a sperm cell [5], so retrieving the flagellar beat patterns, as well as the head trajectories, from image data will help shed new insight on the sperm motility assessment.

In this paper, we present a computational framework designed to track the heads and trace the tails for quantitative analysis of sperm motility. Our framework includes 3 modules: head detection, head tracking, and flagellum tracing. These modules are performed sequentially to obtain the head trajectories and flagellar beat patterns. First, the head detection module detects the sperm heads every 100 frames using a Multiple Birth and Cut (MBC) algorithm [6]. The detections are the inputs to the head tracking module for obtaining the head trajectories and angles of head rotation. We use a block matching method [7] to register the heads in the subsequent images with respect to the positions and angles of those detected heads. This is different from other tracking methods that consider only the head positions. Finally, we propose a flagellum tracing algorithm, based on a Markov chain Monte Carlo (MCMC) sampling method, to obtain the flagellar beat patterns.

\section{METHOD}

\subsection{A Spermatozoon Model}

Modeling of a spermatozoon is essential to extraction and analysis of its movement. We consider a model that represents a spermatozoon as an assembly of a head and a flagellum. Figure 1(a) illustrates this modeling scheme. An ellipse is used to represent the head, and the direction of its major axis is parallel to the direction of sperm movement (the blue arrow). The flagellum is made of a sequence of $N_{s}$ circles. Each circle is tightly connected to its adjacent neighbors and is represented by a radius $(r)$ and an angle $(\theta) . \quad \theta$ is defined as the angle between the straight line connecting the centers of two adjacent circles and the direction of sperm movement. This circle 
representation is shown in Figure 1(b). Hence, given a chain of $N_{s}$ circles, each with $\left(r_{i}, \theta_{i}\right)$, the flagellum length will be $\sum_{i=1}^{N_{s}} 2 r_{i}$.

\subsection{Head Detection}

This module aims at segmenting sperm heads from a 2D image. Our modeling uses an ellipse to represent a head. Hence, a head is represented by 5 parameters, denoted as $(x, y, a, b, \phi)$, where $(x, y)$ are coordinates of the center point, $a$ and $b$ are the lengths of the major and minor axes, respectively, and $\phi$ is the angle between the major axis and the horizontal direction. Using such a representation, the goal of the head detection then becomes to detect an unknown number of ellipse-shaped objects. To achieve this goal, an improved Multiple Birth and Cut (MBC) algorithm based on marked point processes [6] is used. We perform the head detection every 100 frames in order to address the concern that if the sperms are missed by the detection algorithm on the previous frames, they can be detected on a later frame.

\subsection{Head Tracking}

Heads are tracked by co-registering every couple of successive images. For each couple, a subimage is defined around the head in the first image and registered with the second image: this yields the displacement of the head (rotation and translation) that is used in turn to define a sub-image in the second image for the next co-registration. The composition of computed transformations allows to track the head through the sequence. The registration process, built on a block matching technique, iterates two stages in a multi-scale manner [7]. Given a reference image $I$ and a floating image $J$, for each small block in the floating image, the first stage finds the most similar subregion in the reference image based on a similarity criterion. Then, the second stage estimates the global rigid transformation that best describes the obtained correspondences.

\subsection{Flagellum Tracing}

We present a new flagellum tracing method to extract the shapes of flagellar beat from the image sequence in which the sperm head is registered. According to our modeling of a spermatozoon introduced in Section 2.1, the flagellum is made of a sequence of $N_{s}$ circles, $C=\left(c_{1}, \ldots, c_{N_{s}}\right)$, where $c_{i}$ represents the $i$ th circle with parameters $\left(r_{i}, \theta_{i}\right)$. The goal of flagellum tracing is to find the most probable sequence of circles, $\hat{C}$, given the image data $\mathrm{D}$, which is

$$
\hat{\mathrm{C}}=\underset{\mathrm{C}}{\operatorname{argmax}} p(\mathrm{C} \mid \mathrm{D}) \text {. }
$$

Based on the Bayes' rule, Equation (1) can be rewritten as

$$
p(C \mid \mathrm{D}) \propto p(\mathrm{D} \mid C) p(C),
$$

where $p(\mathrm{D} \mid C)$ is the observation likelihood, and $p(C)$ is the prior.

\subsubsection{Circle Prior}

We assume that the prior, $p(C)$, follows a Markov process, that is, circle $c_{i}$ depends only on its previous circle $c_{i-1}$. So the prior can be factorized as

$$
\begin{aligned}
p(C) & =p\left(c_{1}\right) \prod_{i=2}^{N_{s}} p\left(c_{i} \mid c_{i-1}\right) \\
& =p\left(r_{1}, \theta_{1}\right) \prod_{i=2}^{N_{s}} p\left(r_{i}, \theta_{i} \mid r_{i-1}, \theta_{i-i}\right) .
\end{aligned}
$$

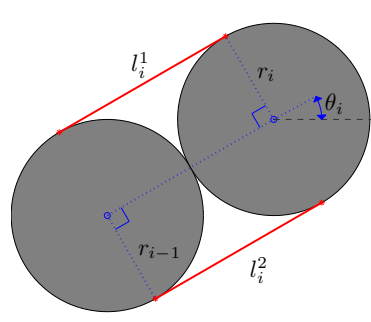

(a)

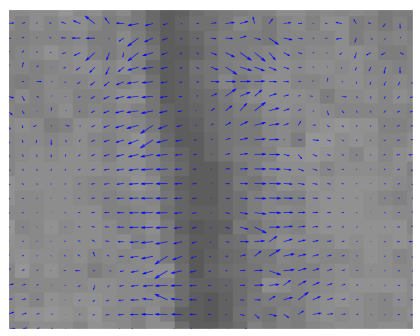

(b)
Fig. 2. Illustration of computation of the observation likelihood. (a) Let $l_{i}^{1}$ and $l_{i}^{2}$ be the two line segments that connect the tangent points of circles $c_{i-1}$ and $c_{i}$. The gradient likelihood considers the normal to the image level lines at each point on lines $l_{i}^{1}$ and $l_{i}^{2}$. Please refer to the text for more detail. (b) The vector fields are diverging from the centerline of a tail (dark) and thus the centerline has positive divergence.

We also assume that the radius $r_{i}$ and orientation $\theta_{i}$ are independent of each other. Then, Equation (3) becomes

$$
p(C)=p\left(r_{1}\right) p\left(\theta_{1}\right) \prod_{i=2}^{N_{s}} p\left(r_{i} \mid r_{i-1}\right) \prod_{i=2}^{N_{s}} p\left(\theta_{i} \mid \theta_{i-1}\right) .
$$

Under such a Markov assumption, the radius $r_{i}$ and orientation $\theta_{i}$ are more likely to be similar to those of the previous circle. Thus, $p\left(r_{i} \mid r_{i-1}\right)$ and $p\left(\theta_{i} \mid \theta_{i-1}\right)$ are modeled using a Gaussian centered around the $r_{i-1}$ and $\theta_{i-1}$, respectively. Mathematically, they are represented as

$$
\left\{\begin{array}{l}
p\left(r_{i} \mid r_{i-1}\right) \propto \exp \left(-\lambda_{r}\left(r_{i}-r_{i-1}\right)^{2}\right) \\
p\left(\theta_{i} \mid \theta_{i-1}\right) \propto \exp \left(-\lambda_{\theta}\left(\theta_{i}-\theta_{i-1}\right)^{2}\right)
\end{array}\right.
$$

where $\lambda_{r}$ and $\lambda_{\theta}$ are constant parameters. For the first circle, its orientation is determined by the detected head, and its radius is estimated from the image data by testing possible radii in a user-defined range. Thus, we set $p\left(r_{1}\right)=1$ and $p\left(\theta_{1}\right)=1$.

\subsubsection{The Observation Likelihood}

The observation model incorporates two constraints: gradient and divergence constraints. Both constraints consider the interaction between pairs of adjacent circles, so we factorize $p(\mathrm{D} \mid \mathrm{C})$ as

$$
p(\mathrm{D} \mid \mathrm{C})=\prod_{i=2}^{N_{s}} p_{g}\left(\mathrm{D} \mid c_{i-1}, c_{i}\right) \prod_{i=2}^{N_{s}} p_{d}\left(\mathrm{D} \mid c_{i-1}, c_{i}\right),
$$

where $p_{g}(\cdot)$ and $p_{d}(\cdot)$ are the gradient and divergence likelihoods, respectively.

Gradient Constraint Before defining this constraint, we first introduce a contrast invariant energy $G(\cdot)$. It is defined over the normal to image level lines, aiming to well locate the object boundary in microscopy images [6]. Specifically, for two adjacent circles $c_{i-1}$ and $c_{i}$ as shown in Figure 2(a), we extract two line segments, $l_{i}^{1}$ and $l_{i}^{2}$, whose endpoints are the tangent points of the circles. Given these two lines, $G\left(l_{i}^{1}, l_{i}^{2}\right)$ is then defined as

$$
G\left(l_{i}^{1}, l_{i}^{2}\right)=\frac{1}{\left|l_{i}^{1}\right|+\left|l_{i}^{2}\right|} \int\left\langle\frac{\nabla g(x)}{\|\nabla g(x)\|}, \mathrm{n}(x)\right\rangle d x
$$


where $\langle\cdot, \cdot\rangle$ is the dot product, $\mathrm{n}(x)$ denotes the outward normal to the line at location $x, \nabla g(x)$ is the gradient vector, and $\left|l_{i}^{1}\right|$ and $\left|l_{i}^{2}\right|$ are the lengths of lines $l_{i}^{1}$ and $l_{i}^{2}$, respectively. Finally, the likelihood $p_{g}\left(\mathrm{D} \mid c_{i-1}, c_{i}\right)$ is given by

$$
p_{g}\left(\mathrm{D} \mid c_{i-1}, c_{i}\right) \propto \exp \left(\lambda_{g} G\left(l_{i}^{1}, l_{i}^{2}\right)\right),
$$

where $\lambda_{g}$ is a constant parameter.

Divergence Constraint We design this constraint to better align the centers of the circles with the centerline of a tail. The divergence indicates the amount of vector field that is converging to, or diverging from, a given point. If the divergence of the vector field has a positive (negative) value, the region is called source (sink). In our sperm data shown in Figure 2(b), the vector fields are diverging from the centerline of a tail (dark), thus the centerline has positive divergence. Mathematically, the divergence of a vector field $v$ is calculated as $\operatorname{div} \mathrm{v}=\frac{\partial v_{x}}{\partial x}+\frac{\partial v_{y}}{\partial y}$. To define the likelihood, we first obtain the line segment $l_{i}$ that connects the centers of circles $c_{i-1}$ and $c_{i}$, and then compute the mean divergence of this line, $D_{l_{i}}$. The divergence likelihood is given by

$$
p_{d}\left(\mathrm{D} \mid c_{i-1}, c_{i}\right) \propto \exp \left(\lambda_{d} D_{l_{i}}\right)
$$

where $\lambda_{d}$ is a constant parameter.

\subsubsection{Markov Chain Monte Carlo Inference}

To estimate the maximum a posteriori (MAP) of Equation (1), a MCMC method is applied to sample the solution space by constructing a Markov chain. We use the Metropolis-Hastings algorithm to generate such a chain. For each circle $c_{i}$ to be estimated, we construct a chain of $N_{c}$ samples by starting with an arbitrary initial sample $c_{i}^{1}$ and then iterating for $s=1 \ldots N_{c}-1$ : (a) Propose a new circle candidate $c_{i}^{\prime}$, (b) calculate the acceptance ratio $\alpha=p\left(c_{i}^{\prime} \mid D\right) / p\left(c_{i}^{s} \mid D\right)$, and (c) accept $c_{i}^{\prime}$ with probability $\alpha$ and reject otherwise. For the latter case, $c_{i}^{s}$ is added to the chain. The sample with the highest probability in the chain is selected as the estimated state. Assuming that the length of a flagellum does not change from one image to the next, we estimate both the radii and the orientations of circles in the image where the head is detected, but only estimate the orientations in the subsequent images.

\section{EXPERIMENTAL RESULTS}

\subsection{Data}

We evaluated the performance of our framework using two confocal microscopy image sequences of ram semen samples. The samples were imaged at two distinct conditions: a control one and a high concentration $(50 \mathrm{mM})$ of beta-mercaptoethanol (bME). In both cases, the frame rate was at $200 \mathrm{fps}$. The size of each frame was $1280 \times 1084(x \times y)$ pixels, with a resolution of $0.3 \times 0.3 \mu \mathrm{m}^{2} /$ pixel.

\subsection{Pre-processing}

The raw images contain noise and inhomogeneous background. We performed a background subtraction step on a temporal sliding window basis to remove such artifacts. Let $I_{j}$ denote the $j$ th image. The background image $B_{j}$ is the median of the images between $I_{j-N_{b}}$ and $I_{j+N_{b}}$, that is, $B_{j}=\operatorname{median}\left\{I_{j-N_{b}}, I_{j-N_{b}+1}, \ldots, I_{j+N_{b}}\right\}$, where $N_{b}$ is the number of images before and after the current image $I_{j}$. We set $N_{b}$ to 30 in the experiments. After obtaining the
Table 1. Object-based Evaluation of Detection Results. The numbers of ground truth $(\mathrm{M})$, detected objects $(\mathrm{N})$, correct detections (CD), missed detections (MD), and false alarms (FA), as well as precision (prec) and recall (rec), regarding different parameter settings $(s)$ are given.

\begin{tabular}{cccccccc}
\hline$s$ & $\mathrm{M}$ & $\mathrm{N}$ & $\mathrm{CD}$ & $\mathrm{MD}$ & $\mathrm{FA}$ & $\mathrm{prec}$ & rec \\
\hline-0.5 & 59 & 77 & 52 & 6 & 25 & 0.8814 & 0.6753 \\
-0.6 & 59 & 66 & 52 & 7 & 14 & 0.8814 & 0.7879 \\
-0.7 & 59 & 43 & 38 & 21 & 5 & 0.6441 & 0.8837 \\
\hline
\end{tabular}

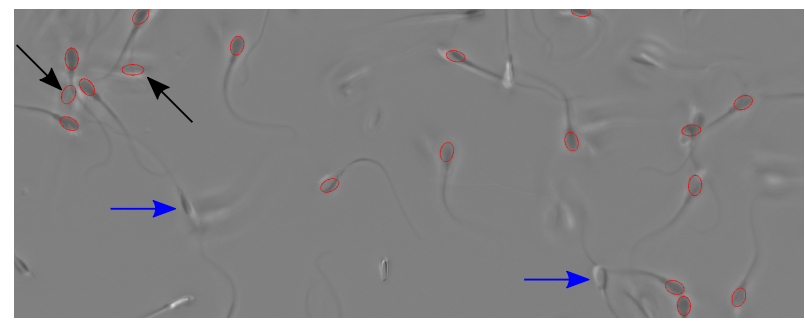

Fig. 3. Part of head detection result $(s=-0.6)$. The contours of the detected heads are depicted in red. The blue (horizontal) arrows indicate the missed detections, and the black (diagonal) arrows point to the false alarms.

background image $B_{j}$, the resulting image $R_{j}$ is obtained by $R_{j}=$ $I_{j}-B_{j}$.

\subsection{Head Detection Results}

The head detection was performed on the background-removed images. Its performance was evaluated by comparing the detection results against manually constructed ground truth. Table 1 summarizes the detection performance regarding different parameter settings in the detection algorithm. Figure 3 shows some detection result.

Using a C++ implementation, the mean computational time for detecting heads on a single frame on a standard laptop with CPU 2.4 $\mathrm{GHz}$ and $8 \mathrm{~GB}$ memory was about 3 mins.

\subsection{Head Tracking Results}

To obtain the head trajectories, for the heads detected every 100 images, we tracked their positions and estimated the angles of head rotation in the subsequent images. As a result, we could obtain, for each detected head, a sequence of images in which the head is still. Figure 4(a) shows a part of the minimum intensity projection (MinIP) of 10 consecutive images from such a sequence. The head trajectory is shown in Figure 4(b), where we can see that the sperm wiggly moves forward with respect to the average path (blue). Figure 4(c) shows the angles of head rotation in the subsequent frames with respect to that in the first frame. As can be seen, the sperm movement involves a periodical behavior.

\subsection{Flagellum Tracing Results}

The parameters for the flagellum tracing were set as $\lambda_{r}=1, \lambda_{\theta}=1$, $\lambda_{g}=1, \lambda_{d}=1$, and the number of samples in the MetropolisHastings algorithm was 200. In the implementation, we used the gradient vector flow (GVF) [8], instead of naive gradient vectors, in the computation of the observation model. Figure 5 shows parts of the tracing results for semen samples imaged at the normal (control) 


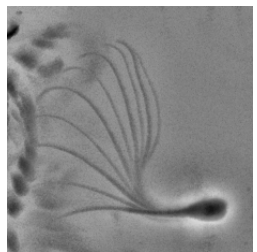

(a) MinIP of $10 \mathrm{imgs}$

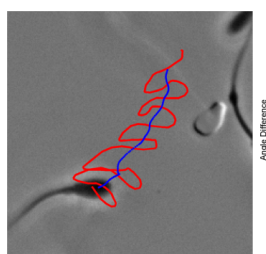

(b) Head trajectory

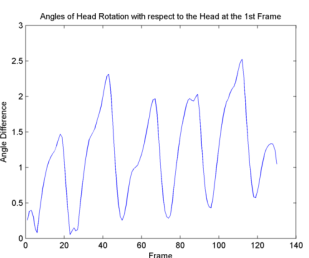

(c) Angles of head rotation
Fig. 4. (a) Minimum Intensity Projection (MinIP) of head-registered images of a sperm, (b) its head trajectory (red) and average path (blue), and (c) angles of head rotation with respect to the head on the first frame.
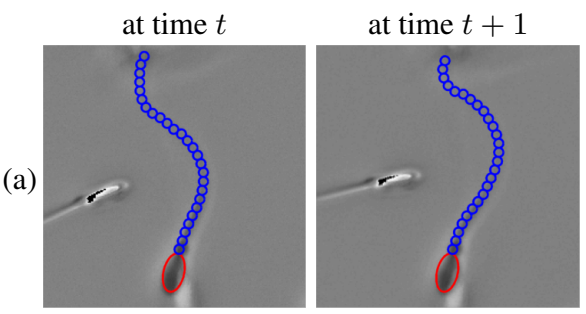

at time $t+3$
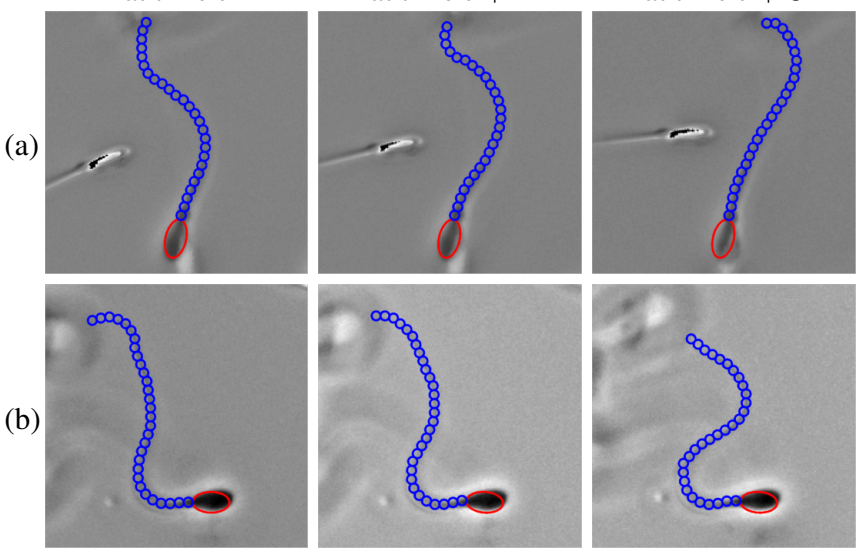

Fig. 5. Flagellum tracing results. (a) Results of a sperm in the control group. (b) Results of a sperm under bME effects. The obtained sequences of circles, along with the ellipses, are overlaid on the images at different times. Visually, the tracing module successfully traces the tails.

and the high concentration of bME conditions. By visual observation, the sequences of circles are lined up well with the flagella in the images, indicating that the proposed method successfully traces the flagella even when the sperms exhibit different beat patterns. More tracing results are shown in Figure 6.

We compared the computer-generated traces against the manual annotations to evaluate the performance of flagellum tracing. The metrics used were missed detection rate (MDR) and false detection rate (FDR) [9]. MDR is the percentage of pixels in the annotation that are more than $d$ pixels away from the computer-generated result; FDR is the percentage of pixels in the generated result that are more than $d$ pixels away from the annotation. We set $d$ to 3 , and the MDR and FDR were $6.12 \%$ and $1.78 \%$, respectively, for 4 selected sperms, each of which was traced for 10 images.

We further compared the sperm behaviors at the normal and bME conditions, which is summarized in Table 2. It shows that the sperms under the effects of a high concentration of bME are of lower beat cross frequency and tail beat frequency and thus move slower.

\section{CONCLUSIONS AND FUTURE WORK}

We presented a framework that performs head detection, head tracking, and flagellum tracing to track the sperm heads and trace the tails for sperm motility analysis. The experimental results demonstrated the effectiveness of our method. In the future, we plan to further (a)
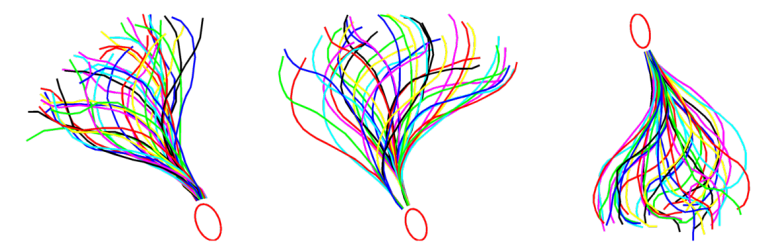

(b)
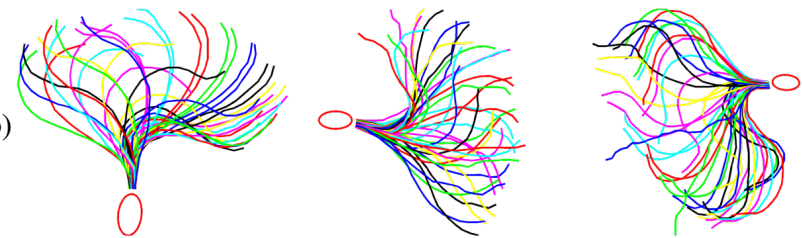

Fig. 6. Flagellar beat patterns of sperms under different conditions. Sperms are (a) at normal condition (control) and (b) at a high concentration of bME. Sperms under the high concentration of bME show larger beat amplitudes. Forty traces for each sperm are shown.

Table 2. Comparison of the Straight Line Velocity (VSL), Beat Cross Frequency (BCF), and Tail Beat Frequency (TBF) of Sperms at the Normal Condition and at the High Concentration of bME. The numbers are represented as mean \pm STD.

\begin{tabular}{cccc}
\hline & VSL $(\mu \mathrm{m} / \mathrm{s})$ & BCF $(\mathrm{Hz})$ & TBF $(\mathrm{Hz})$ \\
\hline Normal & $95.33 \pm 37.56$ & $52.15 \pm 8.67$ & $25.59 \pm 4.30$ \\
bME & $83.61 \pm 21.48$ & $29.17 \pm 5.82$ & $14.58 \pm 2.91$ \\
\hline
\end{tabular}

analyze the head trajectories and flagellar beat patterns to better understand the mechanisms that govern the sperm motility.

\section{REFERENCES}

[1] T. G. Cooper, E. Noonan, S. von Eckardstein, J. Auger, H.W. G. Baker, H. M. Behre, T. B. Haugen, T. Kruger, C. Wang, M. T. Mbizvo, and K. M. Vogelsong, "World Health Organization reference values for human semen characteristics," Human reproduction update, vol. 16, no. 3, pp. 231-245, 2010.

[2] V. R. Nafisi, M. H. Moradi, and M. H. Nasr-Esfahani, "A template matching algorithm for sperm tracking and classification," Physiol. Meas., vol. 26, no. 5, pp. 639-651, 2005.

[3] L. Sørensen, J. Østergaard, P. Johansen, and M. de Bruijne, "Multiobject tracking of human spermatozoa," in Proc. SPIE, Medical Imaging: Image Processing, 2008, vol. 6914, p. 69142C.

[4] J. Liu, C. Leung, Z. Lu, and Y. Sun, "Quantitative analysis of locomotive behavior of human sperm head and tail," IEEE Trans. Biomed. Engineering, vol. 60, no. 2, pp. 390-396, 2013.

[5] B. M. Friedrich, I. H. Riedel-Kruse, J. Howard, and F. Jülicher, "Highprecision tracking of sperm swimming fine structure provides strong test of resistive force theory," J. Experimental Biology, vol. 213, no. 8, pp. 1226-1234, 2010.

[6] E. Soubies, P. Weiss, and X. Descombes, "A 3D segmentation algorithm for ellipsoidal shapes- Application to nuclei extraction," in Proc. Int'l Conf. Pattern Recognition Applications and Methods, 2013, pp. 97105.

[7] S. Ourselin, A. Roche, S. Prima, and N. Ayache, "Block matching: A general framework to improve robustness of rigid registration of medical images," in Medical Image Computing and Computer-Assisted Intervention. 2000, vol. 1935 of LNCS, pp. 557-566, Springer.

[8] C. Xu and J. L. Prince, "Snakes, shapes, and gradient vector flow," IEEE Trans. Image Processing, vol. 7, no. 3, pp. 359-369, 1998.

[9] N. Honnorat, R. Vaillant, J. S. Duncan, and N. Paragios, "Curvilinear structures extraction in cluttered bioimaging data with discrete optimization methods," in Proc. IEEE Int'l Symp. Biomedical Imaging, 2011, pp. 1353-1357. 